\title{
Beneficios del descanso peritoneal
}

\section{Jesús Lucas Martín Espejo, Francisco Cirera Segura, Francisco Barbosa Martín, Carmina Nieto Romero, Eva María Calderón Márquez, Álvaro Pérez Baena}

UGC Nefro-Urológica, Hospitales Universitarios Virgen del Rocío. Sevilla. España

\section{Resumen}

Introducción: Valorar si el descanso peritoneal, de al menos un día semanal, ha supuesto mejoras en la rehabilitación de los pacientes y el impacto clínico que puede suponer. Nos planteamos además cuantificar el beneficio económico.

Material y Método: Se realizó un estudio longitudinal, retrospectivo, descriptivo.

De los pacientes atendidos en nuestra unidad desde el 1 de enero de 2014 al 31 de diciembre de 2014, se estudiaron los pacientes que cumplieran todo el año de seguimiento y que no procedieran de otras técnicas de depuración como la hemodiálisis o trasplante renal.

La muestra de estudio estuvo compuesta por 40 pacientes, de los que 21 tuvieron prescrito al menos 1 día semanal de descanso peritoneal y 19 pacientes sin descanso.

Se recogieron las siguientes variables al inicio del periodo de estudio y a los 12 meses:

Variables demográficas, variables relacionadas con el estado de volumen, datos clínicos relacionados con la técnica, se pasó una encuesta de satisfacción a todos los pacientes estudiados, y se recogieron los importes del coste mensual de cada tipo de tratamiento.

Resultados: Las diferencias iniciales entre los grupos, eran esperables ya que son los valores en los cuales se ha basado la prescripción del día de descanso. La exposición a la glucosa, al comienzo y al final fue menor en el grupo con descanso. Al año, no se encontró diferencias en los parámetros estudiados. Una encuesta de satisfacción nos reveló a que dedican los pacientes el día de descanso y los que no lo tienen a que lo dedicarían.

Correspondencia:

Jesús Lucas Martín Espejo

C/ Gólgota 16-2 .41007 Sevilla

E-mail: jlucasmartin@hotmail.com
Conclusiones: El descanso peritoneal no ha supuesto una peor evolución de los parámetros clínicos estudiados al año de seguimiento, cumpliendo las recomendaciones de adecuación de diálisis de las guías clínicas. La exposición a la glucosa, aun sin llegar a ser significativa por la duración del estudio, ha sido menor en el grupo con descanso.

El día de descanso ha facilitado a los pacientes mejorar su rehabilitación social principalmente.

Los días de descanso han supuesto un ahorro de al menos $13,33 \%$ en la factura mensual.

PALABRAS CLAVE

- DIÁLISIS PERITONEAL

- DESCANSO PERITONEAL

- REHABILITACIÓN

\section{Benefits of peritoneal rest}

\section{Abstract}

Introduction: To assess whether the peritoneal rest at least one day a week has led to improvements in the rehabilitation of patients, and the clinical impact that can be achieved. We also propose to quantify the economic benefit.

Material and methods: A longitudinal, retrospective, descriptive study was conducted.

Of those patients seen in our unit from 1 January 2014 to 31 December 2014, patients who fulfilled all the monitoring year and that at the same time they were not subjected to other purification techniques such as dialysis or transplantation kidney were studied.

The study sample was composed of 40 patients, of whom 21 had prescribed at least 1 weekly day of peritoneal rest, and 19 patients without rest. 
The following variables at baseline and 12 months of the study period were recorded: Demographic variables, variables related to volume status, clinical data related to the technique and data related to a satisfaction survey, as well as the amounts of the monthly cost of each type of treatment.

Resultados: The initial differences between the groups were expected since they are the values on which is based the prescription of the rest day. Exposure to glucose at the beginning and the end was lower in the group with rest. At one year, no differences in the studied parameters were found. A satisfaction survey revealed to us the activity that patients spend the rest day, as well as the activity that would perform those who not rest.

Conclusiones: The peritoneal rest has not meant a worse outcome of the clinical parameters studied at one year, fulfilling the recommendations of dialysis adequacy of the guidelines. Although not statistically significant for the duration of the study, exposure to glucose was lower in the group with rest. The rest day has mainly provided to patients to improve their social rehabilitation. Rest days have generated savings on the monthly bill of $13.33 \%$.

\section{PALABRAS CLAVE}

- PERITONEAL DIALYSIS

- PERITONEAL REST

- REHABILITATION

\section{Introducción}

La diálisis peritoneal (DP) es una técnica de depuración extrarrenal continua en la cual la membrana peritoneal se somete a significativas alteraciones patológicas durante la exposición a largo plazo al fluido convencional de diálisis peritoneal.

Normalmente este tratamiento es realizado por los pacientes varias veces al día todos los días de la semana, variando su frecuencia en función de la sintomatología urémica, la función renal residual (FRR) y los parámetros analíticos.

El hecho de que algunos de los pacientes conserven algo de FRR y un volumen de diuresis significativo, que se asocia a una mejor supervivencia ${ }^{1}$, favorece que la dosis de diálisis que tengamos que administrar pueda ser menor que en aquellos pacientes que no la conservan.

Algunas medidas que podemos tomar para mantener la FRR, así como para aumentar la supervivencia a largo plazo de la técnica e incluso disminuir el riesgo relativo de muerte, son el uso de líquido biocompatibles e Icodextrina (para la conservación de la membrana peritoneal) o mantener un período de "reposo" peritoneal que ha demostrado restaurar la capacidad de ultrafiltración peritoneal ${ }^{1,2,3}$.

Las características del tratamiento con DP pueden impedir, en ocasiones, una adecuada rehabilitación social de los pacientes. Poder contar con algún tiempo libre del tratamiento puede facilitarles realizar actividades que habrían tenido que abandonar al tener que realizar el tratamiento diariamente.

Por estos motivos nos planteamos como objetivo del estudio valorar si el descanso peritoneal, de al menos un día semanal, ha supuesto mejoras en la rehabilitación de los pacientes y el impacto clínico que puede suponer. Como objetivo secundario nos planteamos cuantificar el beneficio económico del descanso peritoneal.

\section{Material y método}

Se realizó un estudio retrospectivo comparativo Iongitudinal.

Se incluyeron en el estudio los pacientes atendidos desde el 1 enero de 2014 a 31 de diciembre de 2014, lo que supuso una población de 95 pacientes. Seleccionamos a los pacientes tras aplicar los siguientes criterios de inclusión:

- Pacientes incidentes en DP no procedentes de otras técnicas (hemodiálisis o trasplante renal).

- Paciente que cumplieran un año de seguimiento en los grupos que a continuación describiremos.

La muestra para el estudio fue de 40 pacientes: 21 pacientes con descanso peritoneal de al menos 1 día semanal (8 en diálisis peritoneal automática (DPA) y 13 diálisis peritoneal continua ambulatoria (DPCA)) y 19 pacientes sin descanso peritoneal (3 en DPA y 16 en DPCA).

Se recogieron las siguientes variables de la historia digital de los pacientes; al inicio del estudio, así como a los 12 meses de seguimiento:

1. Variables demográficas: edad, género, patología de base de la enfermedad renal crónica, técnica de diálisis.

2. Variables relacionadas con el estado de volumen: diuresis de 24 horas, peso, tensión arterial sistólica y diastólica. 
3. Variables relacionadas con la técnica: Creatinina, filtrado glomerular (eGFR por Padequest ${ }^{\circledR}$ ), fósforo, hematocrito y hemoglobina. Kt/V peritoneal, renal y total, aclaramiento de creatinina (Padequest ${ }^{\circledR}$ ), exposición a la glucosa ${ }^{4}$ e infecciones peritoneales.

4. Encuesta de satisfacción para valorar el posible beneficio social.

5. Coste mensual de cada tratamiento en cada modalidad de DP.

\section{Resultados}

Se estudiaron un total de 40 pacientes, con una edad media 58,95 $\pm 16,76$ años, siendo hombres el $52,5 \%(n=21)$. La etiología de la enfermedad renal crónica (ERC) más frecuente fue de origen no filiado en el $37,5 \%(n=15)$, la nefropatía diabética en segundo lugar $17,5 \%(n=7)$, seguidas de las glomerulonefritis y las de etiología vascular, ambas con un $10 \%$ $(n=4)$ cada una.

El $72,5 \%(n=29)$ de los pacientes estaba en DPCA y el $27,5 \%(n=11)$ en DPA.

Se hicieron los dos grupos de estudio siguiendo los criterios establecidos, donde el grupo sin descanso peritoneal estaba compuesto por 19 pacientes y el grupo con al menos un día de descanso peritoneal por 21 pacientes y se analizaron sus características basales. (Tabla $\mathbf{1}$ ).
Tabla 2. Evolución de los parámetros estudiados en ambos grupos al año de seguimiento

\begin{tabular}{|l|c|c|c|}
\hline & Grupo sin descanso & Grupo con descanso & $\mathbf{p}$ \\
\hline Diuresis (cc) & $217,10 \pm 500,56$ & $138,09 \pm 499,22$ & $0,751^{2}$ \\
\hline Peso (Kg.) & $-2,28 \pm 3,32$ & $-1,81 \pm 5,27$ & $0,749^{1}$ \\
\hline P.A. sistólica (mm de Hg) & $22,84 \pm 32,64$ & $1,52 \pm 23,15$ & $0,045^{2}$ \\
\hline P.A. diastólica (mm de Hg) & $8,15 \pm 17,41$ & $0,42 \pm 16,54$ & $0,158^{1}$ \\
\hline eGFR (mL/min) & $1,20 \pm 1,35$ & $5,28 \pm 2,74$ & $0,222^{2}$ \\
\hline Creatinina (mg/dL) & $-0,15 \pm 1,87$ & $-1,09 \pm 1,49$ & $0,08^{1}$ \\
\hline Ktv peritoneal & $-0,15 \pm 1,86$ & $-0,13 \pm 0,22$ & $0,850^{1}$ \\
\hline Kt/V renal & $0,26 \pm 0,34$ & $1,03 \pm 0,51$ & $0,635^{2}$ \\
\hline Kt/V total & $-0,03 \pm 0,52$ & $0,05 \pm 0,49$ & 0,6241 \\
\hline A. Creatinina (litro/sem) & $-2,06 \pm 17,40$ & $6,78 \pm 23,81$ & $0,192^{1}$ \\
\hline Fósforo (mg/dL) & $4,76 \pm 0,79$ & $4,68 \pm 0,61$ & $0,473^{2}$ \\
\hline Hematocrito (\%) & $34,37 \pm 4,89$ & $35,21 \pm 3,33$ & $0,498^{2}$ \\
\hline Hemoglobina (g/dL) & $0,35 \pm 1,63$ & $0,48 \pm 1,27$ & $0,766^{1}$ \\
\hline
\end{tabular}

${ }^{1} \mathrm{~T}$ de Student. ${ }^{2} \mathrm{U}$ de Mann Whitney. P.A: presión arterial. eGFR: estimación del filtrado glomerular.
Tabla 1. Características basales de los grupos estudiados

\begin{tabular}{|l|c|c|c|}
\hline & Grupo sin descanso & Grupo con descanso & p \\
\hline Edad (años) & $59,42 \pm 18,68$ & $58,62 \pm 15,29$ & $0,868^{1}$ \\
\hline Género (hombres) & $52,6 \%(\mathrm{n}=10)$ & $52,4 \%(\mathrm{n}=11)$ & $0,987^{2}$ \\
\hline Diuresis (cc.) & $528,94 \pm 678,5$ & $1330,95 \pm 749,74$ & $0,001^{3}$ \\
\hline Peso (Kg.) & $75,76 \pm 17,73$ & $75,55 \pm 15,05$ & $0,968^{1}$ \\
\hline P.A.Sistólica (mm de Hg) & $132,73 \pm 21,84$ & $138,66 \pm 19,16$ & $0,366^{1}$ \\
\hline P.A.Diastólica (mm de Hg) & $75,26 \pm 15,77$ & $77,48 \pm 12,54$ & $0,655^{3}$ \\
\hline eGFR (mL/min) & $1,62 \pm 1,70$ & $6,51 \pm 2,32$ & $<0,001^{3}$ \\
\hline Creatinina (mg/dL) & $8,88 \pm 2,88$ & $6,63 \pm 2,19$ & $0,003^{1}$ \\
\hline Kt/v peritoneal & $1,60 \pm 0,37$ & $1,35 \pm 0,26$ & $0,017^{1}$ \\
\hline Kt/v renal & $0,34 \pm 0,36$ & $1,21 \pm 0,42$ & $<0,001^{1}$ \\
\hline Kt/v total & $1,94 \pm 0,39$ & $2,56 \pm 0,45$ & $<0,001^{1}$ \\
\hline A.Creatinina (L/sem.) & $58,65 \pm 13,19$ & $100,40 \pm 18,79$ & $<0,001^{1}$ \\
\hline Fosforo (mg/dL) & $5,21 \pm 1,17$ & $4,97 \pm 1,40$ & $0,329^{2}$ \\
\hline Hematocrito (\%) & $33,68 \pm 8,49$ & $35,86 \pm 3,47$ & $0,734^{3}$ \\
\hline Hemoglobina (g/dL) & $11,41 \pm 1,32$ & $11,69 \pm 1,19$ & $0,493^{1}$ \\
\hline
\end{tabular}

${ }^{1} \mathrm{~T}$ de Student. $b^{2} \mathrm{Chi}$ cuadrado. ${ }^{3} \mathrm{U}$ de Mann Whitney. P.A: presión arterial. eGFR: estimación del filtrado glomerular.
No hubo diferencias iniciales respecto a la etiología de la ERC en ambos grupos $(p=0,115)$. Respecto a las diferencias iniciales entre los grupos, eran esperables ya que son los valores en los cuales se ha basado la prescripción del día de descanso como es el caso de la diuresis, creatinina y el filtrado glomerular, o como resultado de la misma prescripción: Kt/V y aclaramientos. Posteriormente se analizaron su evolución y su significación.

La exposición diaria de glucosa se calculó como el producto del volumen y la concentración de glucosa para todos los intercambios diarios.
El promedio de la exposición a la glucosa al comienzo del estudio en el grupo con descanso fue de $132,99 \pm 76,61 \mathrm{~g}$. frente a $143,01 \pm 92,93 \mathrm{~g}$. $(p=0,860)$ en el grupo sin descanso y al final del estudio fueron $137,42 \pm 80,48 \mathrm{~g}$. frente a $168,76 \pm 104,46 \mathrm{~g}$. para el grupo sin descanso $(p=0,28)$.

Analizamos los mismos datos al año de seguimiento, para ver si los parámetros estudiados variaban de forma significativa en función de tener un periodo de descanso. No se encontró ninguna diferencia en la evolución de los parámetros estudiados, exceptuando en la presión arterial sistólica, en el que grupo con descanso peritoneal había sufrido un incremento significativamente menor. (Tabla 2). 
A pesar del día de descanso, el $\mathrm{Kt} / \mathrm{v}$ total medio al año de seguimiento era de $2,51 \pm 0,5$, y el aclaramiento de creatinina de 93,62 $\pm 23,95$ litros semanales.

Hubo 3 pacientes con cuatro infecciones peritoneales en el grupo con días de descanso, frente a 5 pacientes con 9 infecciones peritoneales (uno de ellos con 4 ) en el grupo sin descanso.

Se realizó una pequeña encuesta a los pacientes para saber si estaban satisfechos con el día de descanso, a qué lo dedicaban y si suponía una liberación del tratamiento en su vida. Igualmente se preguntó a los pacientes que no tenían prescrito el descanso peritoneal para saber a qué hubiesen dedicado ese tiempo. Como resultado de la encuesta, el $100 \%$ de los pacientes comentaron estar satisfecho al poder tener uno o más días de descanso, el 33,3\% $(n=7)$ de los pacientes lo utilizaban para hacer pequeños viajes por la comunidad. Otro $33,3 \%(n=7)$ de los pacientes lo utilizaban para actividades lúdicas. El 19,04\% $(n=4)$ continuaban con sus actividades habituales. El $9,52 \%(n=2)$ para hacer ejercicio al aire libre y el $4,76 \%(n=1)$ para actividades diversas. El $76,19 \%$ $(n=16)$ de los pacientes comentaron sentirse liberado de la diálisis el día de descanso, mientras que el $23,8 \%(n=5)$ comentaron que no.

A los pacientes sin descanso se les preguntó a que lo dedicarían si lo tuviesen. El 36,84\% $(n=7)$ realizarían sus actividades habituales de forma más relajada. El $21,05 \%(n=4)$ no sabían a que lo dedicarían. El $10,52 \%(n=2)$ realizarían actividades de ocio. El $5,26 \%(n=1)$ lo utilizarían para realizar pequeños viajes. El 5,26\% $(n=1)$ para dormir más. El 5,26\% $(n=1)$ dedicaría el tiempo sin tratamiento a sus actividades laborales. Un $15,78 \%(n=3)$ de los pacientes fallecieron antes de poder pasarle la encuesta por motivo ajenos a la técnica.

El día de descanso de los pacientes supuso un ahorro económico calculado de al menos el $13,33 \%$ del precio total del tratamiento, lo que supondría un ahorro de $214 € /$ mes para los pacientes en DPCA con bicarbonato y $232 € /$ mes para pacientes en DPA con volúmenes inferiores a $15 \mathrm{~L}$. Estas cifras se duplicarían o triplicarían en aquellos pacientes que descansaban más de un día.

\section{Discusión}

Los cambios fisiopatológicos que se desarrollan con el tiempo en la membrana peritoneal se deben a varios factores, entre ellos la exposición a las soluciones de DP, sobre todo si son líquidos no biocompatibles y a la respuesta presencial del catéter peritoneal ${ }^{1,8}$. Zhen y col, sugieren que el descanso peritoneal corto puede mejorar la capacidad de ultrafiltración y por lo tanto disminuir el uso de soluciones hipertónicas³.

Otros estudios publicados refieren que el descanso peritoneal parece mejorar la ultrafiltración por la disminución del engrosamiento peritoneal, la tasa de absorción de fluido, la absorción de líquido linfático de la cavidad peritoneal y la hiperpermeabilidad a la glucosa ${ }^{3,6}$.

En un estudio in vitro, se observó que las células mesoteliales peritoneales humanas pueden recuperarse del estrés del líquido peritoneal con un descanso de 24 horas $^{5}$. La causa de fallo de ultrafiltración peritoneal en algunos pacientes en DPCA sigue siendo desconocida, pero puede estar relacionada con la incapacidad del mesotelio a la regeneración de la capacidad después de agresiones agudas 0 crónicas $^{8}$.

Todos estos hallazgos parecen indicar que es recomendable dejar descansar el peritoneo de las agresiones que suponen los líquidos de diálisis peritoneal, siempre y cuando se adecuen individualmente las dosis de diálisis administradas, siguiendo las guías de práctica clínica ${ }^{9,10}$.

En nuestro estudio no encontramos ninguna diferencia en la evolución de los parámetros estudiados al año de seguimiento, excepto en la presión sistólica donde se encontró un menor incremento en el grupo con descanso. Además, el filtrado glomerular, el Kt/V total y el aclaramiento de creatinina, habían aumentado en el grupo con descanso al final del estudio, aunque sin significación estadística, frente al grupo sin descanso donde estos parámetros habían disminuido. El resto de los parámetros siguen cumpliendo las recomendaciones de las guías de prácticas clínica ${ }^{9,10}$ aún teniendo un día de descanso peritoneal.

A pesar de que el grupo con descanso peritoneal presentaba un mayor número de pacientes en DPA, en los que los volúmenes suelen ser más altos y a priori la exposición a la glucosa es mayor, no han presentado una mayor exposición a la glucosa ni al principio ni al final del estudio, debido al día de descanso. 
El grupo sin descanso presentó un número mayor de infecciones peritoneales, pudiendo estar relacionado con la realización de un mayor número de intercambios a lo largo del año de estudio, circunstancia esta que predispone al riesgo de infección.

El $80 \%(n=17)$ de los pacientes que tenían días de descanso, se han visto beneficiados al poder realizar actividades diversas (viajes, lúdicas, ejercicios, etc.) que según los mismos pacientes, han sido viables al disponer de un periodo más largo de tiempo libre de la DP y no tener que trasladar el material en los desplazamientos. Un $19 \%$ de los pacientes no utilizarían este tiempo para nada fuera de sus actividades habituales, aunque algunos de estos pacientes eran los que disponían de más de un día de descanso.

En los pacientes sin descanso llama la atención que el $36,84 \%$ lo utilizarían para hacer la rutina habitual de forma más relajada, lo que nos hace pensar que el hecho de estar pendiente de los horarios de los cambios puede intranquilizar a los pacientes en su rutina diaria.

A partir del resultado de las encuestas y resumiendo los hallazgos, consideramos beneficioso para la rehabilitación social de los pacientes el descanso peritoneal.

La diálisis es la terapia crónica más cara. Su coste es entre 6 y 7 veces mayor que el tratamiento de un paciente con SIDA y entre 30 o 40 veces mayor que el de la EPOC ${ }^{7}$. El descanso peritoneal ha supuesto un ahorro económico de al menos un $13,33 \%$ del coste mensual del tratamiento, resultando coste eficiente al sistema sanitario.

\section{Conclusiones}

El descanso peritoneal no ha supuesto una peor evolución de los parámetros clínicos estudiados al año de seguimiento, y los pacientes cumplieron las recomendaciones de adecuación de diálisis de las guías de práctica clínica. Por lo que pensamos que no ha influido negativamente que los pacientes tuviesen al menos un día de descanso peritoneal.

La exposición a la glucosa, aun sin llegar a ser significativa por la duración del estudio, ha sido menor en el grupo de descanso, con los beneficios que ello puede aportar. Aun así, la repercusión del descanso peritoneal sobre la membrana peritoneal, requiere estudios de mayor duración.
El día de descanso ha facilitado a los pacientes mejorar su rehabilitación social principalmente.

Los días de descanso han supuesto un ahorro de al menos $13,33 \%$ en la factura mensual del tratamiento.

Recibido: 7 julio 2015

Revisado: 23 julio 2015

Modificado: 10 agosto 2015

Aceptado: 14 agosto 2015

\section{Bibliografía}

1. Termorshuizen F, Korevaar JC, Dekker FW, van Manen JG, Boeschoten EW, Krediet RT: The relative importance of residual renal function compared with peritoneal clearance for patient survival and quality of life: An analysis of the Netherlands Cooperative Study on the Adequacy of Dialysis (NECOSAD)-2. Am J Kidney Dis 241: 1293-1302, 2003.

2. Kendrich J, TeitelbaumI.Strategies for Improving Long-Term Survival in Peritoneal Dialysis Patients. Clinical Journal of American Society Nephrology, 2010, 5 (6):1123-1131.

3. Zhe $X$, Tian X, Cheng L, Wang T: Effects of peritoneal resting on peritoneal fluid transport kinetics. Perit Dial Int 27: 575-579, 2007.

4. Rocco MV, Jordan JR, Burkart JM. Determination of peritoneal transport characteristics with 24hour dialysate collections: dialysis adequacy and transport test. J Am SocNephrol 1994; 5:1333-8.

5. Tomo T, Okabe E, Matsuyama K, Iwashita T, Yufu K, Nasu M. The effect of peritoneal rest in combination therapy of peritoneal dialysis and hemodialysis: using the cultured human peritoneal mesothelial cell model. J ArtifOrgans 2005; 8:125-9.

6. de Alvaro F, Castro MJ, Dapena F, Bajo MA, Fernandez-Reyes MJ, Romero JR, et al. Peritoneal resting is beneficial in peritoneal hyperpermeability and ultrafiltration failure. AdvPerit Dial 1993; 9:56. 
7. Arrieta J, Rodríguez-Carmona A, Remón C , PérezFontán M, Ortega F, Sánchez Tornero JA, Selgas R. La diálisis peritoneal es la mejor alternativa costeefectiva para la sostenibilidad del tratamiento con diálisis. Nefrología 2011;31(5):505-513.

8. Rodrigues A, Cabrita A, Maia P, Guimaraes S. Peritoneal rest may successfully recover ultrafiltration in patients who develop peritoneal hyperpermeability with time on continuous ambulatory peritoneal dialysis. AdvPerit Dial 2002; 18:78-80.
9. K/DOQI Clinical Practice guidelines forchronic kidney disease: Evaluation, classification, and stratification.Am J KidneyDis 2002;39(2 Suppl 1):S1-266.

10. Guías SEN. Guías de práctica clínica en diálisis peritoneal de la SEN. Nefrología 2006; 26 (4). 